\section{COMPARATIVE IMMUNOLOGY}

\section{Bat adaptive immunity}

PLoS Biology https://doi.org/10.1371/journal. pbio.3000436 (2019)

Bats are important natural reservoirs for detrimental human zoonotic viruses but are themselves generally asymptomatic carriers. In PLoS Biology, Liu and colleagues investigate the contribution that adaptive immunity might provide to the resistance of bats to viruses that are usually highly pathogenic in most other mammals. Looking at the presentation of peptides from four different viruses, including MERS and Ebola viruses, by bat major histocompatibility complex class I, they observe several unusual structural features. Bats have a three- or five-amino acid addition that leads to extension of the $\alpha 1$ helix of major histocompatibility complex class I and tighter insertion of the peptide into the binding groove. The five-amino acid insertion is unique to bats, whereas the three-amino acid insertion is also seen in some marsupials. There is also unusual use of a proline in the terminal amino acids of the bound peptide. Collectively, these features may underpin more-effective adaptive antiviral immunity.

https://doi.org/10.1038/s41590-019-0533-8

\section{VACCINES}

\section{Neutralizing HSV}

Sci. Immunol. 4, eaaw7083 (2019)

Herpes simplex virus type $2(\mathrm{HSV}-2)$ is a common sexually transmitted pathogen that establishes lifelong infection with recurrent episodic genetic lesions and subclinical viral shedding. Although antibody responses can control HSV-2, so far no effective vaccine against this virus has been developed. In Science Immunology, Awasthi et al. describe a preclinical study testing the efficacy of trivalent protein or mRNA vaccine formulations designed to neutralize HSV-2 and prevent subclinical viral replication and shedding. Both vaccine formulations target viral glycoproteins gC2, gD2 and gE2, which are responsible for viral entry and immunoevasion. Both vaccines also elicit neutralizing antibody responses to HSV-2 as well as to HSV-1. While the trivalent protein-subunit vaccine against HSV-2 provides partial protection to infected mouse and guinea pigs, lipid nanoparticles containing modified mRNAs (mRNALNPs) encoding the viral glycoproteins give superior protection against intravaginal viral challenge, prevent HSV-2 replication in dorsal root ganglion cells and block viral shedding. These findings offer hope for the development of effective mRNA-LNP vaccines for humans.

https://doi.org/10.1038/s41590-019-0534-7

\section{AUTOIMMUNITY}

\section{ZF Pathogenic antibodies}

$$
\text { Nature 574, 122-126 (2019) }
$$

\section{Pathogenic autoantibodies contribute to} various immune-mediated diseases. Whether distinct immune-mediated diseases in humans share similar features that shape their circulating B cell pool and hence their $B$ cell antigen receptor (BCR) repertoires has remained unknown. In Nature, Smith and colleagues report a comparative analysis of circulating BCR repertoires from newly diagnosed patients across six
After injury, cardiac fibroblasts induce cardiac fibrosis, which is an important factor in the progression of cardiac disease. In Nature, Epstein and colleagues use T cell immunotherapy to limit cardiac fibrosis in a mouse model of cardiac injury. Mice that express ovalbumin peptide in activated cardiac fibroblasts, but not in quiescent cardiac fibroblasts, and have received ovalbumin-specific CD8 ${ }^{+}$OT-I T cells develop less cardiac fibrosis than do their counterparts not given T cell transfer. The authors show that the endogenous glycoprotein FAP is not expressed in healthy hearts or other tissues in mice and humans but is expressed in cardiac fibroblasts in patients with cardiomyopathy. Injured mice given transfer of T cells engineered to express a chimeric antigen receptor (CAR T cells) directed against FAP have less fibrosis than that of injured mice that did not receive CAR T cells, and also show partial restoration of heart function. FAP CAR $T$ cell therapy shows no evidence of systemic or cardiac toxicity and does not delay wound healing in a model of skin injury. distinct autoimmune diseases. Among the parameters analyzed are frequency of the gene cluster encoding the immunoglobulin heavy-chain variable region, and its isotype and diversity, as well as clonal size and inferred relationships between naive $B$ cells and antigen-experienced B cells. The BCR repertoires of patients with systemic lupus erythematosus exhibit the expected differences relative to those of healthy control subjects but, surprisingly, so do the BCR repertoires of patients with Crohn's disease or eosinophilic granulomatosis, despite the fact that these diseases have not been associated with pathogenic B cells previously. The authors find increased circulating titers of immunoglobulin A ( $\operatorname{IgA}$ ) or $\operatorname{IgE}$ or both $\operatorname{IgA}$ and $\operatorname{IgE}$ in all three diseases. Importantly, they note differences in patient responses to anti-B cell therapies. These features can provide insights into strategies for treating patients with these diseases.

https://doi.org/10.1038/s41590-019-0535-6

\section{MICROBIOTA}

\section{Gut ecology}

Cell https://doi.org/10.1016/j.cell.2019.08.011 (2019)

Fiber-based dietary interventions can sustain beneficial microbiota. In Cell, Gordon and colleagues characterize the dietary fibers that selectively increase the fitness of the Bacteroides species in the human microbiota. In germ-free mice colonized with consortia of bacterial strains from a lean human donor and fed lowfiber diets supplemented with combinations of fiber preparations from diverse plant sources, particular fiber types have pronounced effects on specific Bacteroides species: pea fiber expands B. thetaiotaomicron; barley bran expands B. ovatus; and citrus pectin expands B. cellulosilyticus. In the respective Bacteroides species, most proteins induced are encoded by polysaccharide utilization loci and are involved in metabolism of the most abundant polysaccharide in these fibers (arabinan in pea fiber, and galacturonan in citrus pectin). $B$. cellulosilyticus and $B$. vulgatus compete for arabinan and galacturonan, with the former always suppressing the latter. B. cellulosilyticus and B. ovatus compete for arabinoxylan in the basal low-fiber diet, but the latter displays metabolic flexibility to mitigate competition. These results can inform efforts to enrich beneficial microbiota.

https://doi.org/10.1038/s41590-019-0536-5 